\title{
Monte Carlo Simulation of Exciton Bimolecular Annihilation Dynamics in Supramolecular Semiconductor Architectures ${ }^{\dagger}$
}

\author{
Clément Daniel, Sebastian Westenhoff, François Makereel, and Richard H. Friend \\ Cavendish Laboratory, University of Cambridge, Madingley Road, Cambridge CB3 OHE, United Kingdom
}

\author{
David Beljonne \\ Chemistry of Novel Materials, University of Mons-Hainaut, Place du Parc 20, B-7000 Mons, Belgium
}

\author{
Laura M. Herz \\ Clarendon Laboratory, Oxford University, Parks Road, Oxford OX1 3PU, United Kingdom
}

\begin{abstract}
Carlos Silva*
Département de Physique \& Regroupement Québécois sur les Matériaux de Pointe, Université de Montréal, c.p. 6128, succ. Centre-Ville, Montréal, Québec H3C 3J7, Canada
\end{abstract}

Received: December 17, 2005; In Final Form: April 23, 2007

\begin{abstract}
We present a simulation of exciton dynamics in supramolecular assemblies of an oligo-p-phenylenevinylene derivative monofunctionalised with a quadruple hydrogen-bonding group (MOPV). MOPV molecules form helical stacks in dodecane solution through solvophobic and $\pi-\pi$ interactions with thermotropic reversibility. We apply a model of incoherent excitation hopping using a Monte Carlo scheme to extract microscopic physical quantities relevant to energy diffusion and bimolecular annihilation processes within isolated nanostructures. We compare the simulation to ultrafast spectroscopic data, namely photoinduced absorption transients at various excitation fluences, their polarization anisotropy, and the dynamic photoluminescence red-shift. We observe that energy diffusion and bimolecular annihilation processes can be described with the same microscopic model based on a Förster-like model that takes into account the spatial extent of the excited state; these two processes are interconnected via the same underlying physics. We extract a high diffusion coefficient $\left(\sim 0.08 \mathrm{~cm}^{2} \mathrm{~s}^{-1}\right)$ over the first few picoseconds following excitation, which plays an important role in dictating the bimolecular annihilation dynamics.
\end{abstract}

\section{Introduction}

Polymeric semiconductors are an exciting class of materials that are now finding commercial applications in light-emitting diodes (LEDs) for electroluminescent displays ${ }^{1-4}$ and show promise in other applications such as in photovoltaic diodes (PVDs). ${ }^{5-10}$ It is now widely accepted that the primary photoexcitations in phenylene-based $\pi$-conjugated polymers are singlet excitons, and that these migrate within the bulk polymer phase by incoherent stepwise hops that are mediated by resonance energy transfer (RET). Exiton migration dynamics play an important role in the optoelectronic properties of these materials. ${ }^{11}$ For example, these are essential for the efficient operation of polymer-based PVDs, in which the light absorbed in the bulk polymer must be transported to a charge-transfer heterojunction where efficient charge separation occurs. ${ }^{12-15}$ Furthermore, exciton diffusion away from such heterojunctions is essential after charge recombination in LEDs to prevent retrapping and possible charge separation. ${ }^{16,17}$ In this paper, we model exciton dynamics, derived from ultrafast spectroscopic measurements, within isolated semiconductor nanostructures

$\dagger$ Originally submitted for the "Robert J. Silbey Festschrift", published as the September 28, 2006, issue of J. Phys. Chem. B (Vol. 110, No. 38).

* Corresponding author. E-mail address: carlos.silva@umontreal.ca. applying Monte Carlo simulations of RET processes. We explore exciton bimolecular signatures as a probe of such dynamics.

The appropriate description of RET in molecular materials is dependent on the strength of intermolecular electronic coupling with respect to intramolecular exciton-phonon coupling, which in turn depends on the intermolecular separation among other factors. In the weak coupling limit, which is applicable in most situations relevant to polymeric semiconductors, strong vibronic coupling and energetic disorder localize the exciton on one chromophore and subsequent transport occurs by incoherent hopping processes between chromophores. ${ }^{18-22,24-26}$ The RET rate constant is then described within a golden-rule formalism as

$$
k=\frac{2 \pi}{\hbar} I_{\mathrm{DA}} V_{\mathrm{DA}}^{2}
$$

where $I_{\mathrm{DA}}$ is the overlap integral between the donor photoluminescence spectrum and the acceptor absorption spectrum and $V_{\mathrm{DA}}$ is the donor-acceptor Coulomb coupling. The transfer rate is proportional to the product of the interchromophore coupling and an overlap integral with homogeneous broadening and the vibronic progressions. If the exchange interaction is neglected, $V_{\text {DA }}$ becomes the electrostatic interaction between the electronic transition densities associated with the donor and acceptor 


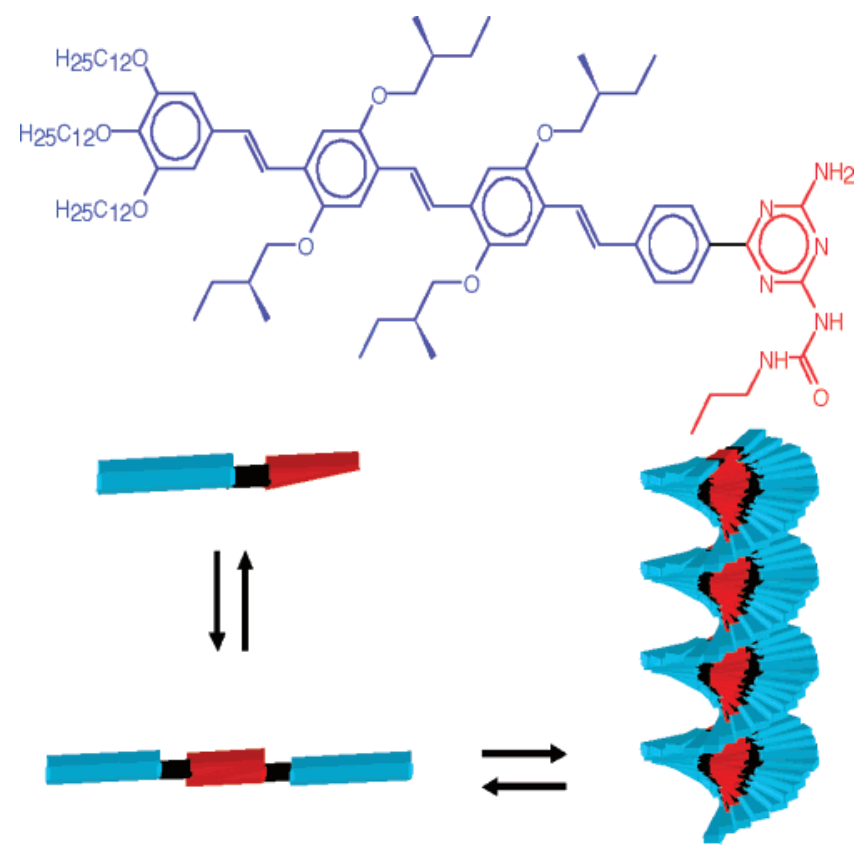

Figure 1. Molecular structure of the MOPV material used in this paper. Also shown is a schematic representation of the supramolecular selfassembly of MOPV in dodecane solution. MOPV molecules dimersise by quadrupolar hydrogen bonding, followed by spontaneous selfassembly into chiral stacks due to solvophobic and $\pi-\pi$ interactions. The self-assembly is thermotropically reversible.

molecules. ${ }^{27}$ In the approach of Förster, the point-dipole approximation allows $k$ to be conveniently extracted from experimentally available quantities but fails when the interchromophore distance is comparable to the size of the localized exciton. ${ }^{21-23}$ In this case, a suitable approach is the so-called line-dipole approximation where the molecular transition dipoles are subdivided in fragmented dipoles. ${ }^{24}$ The electronic coupling is then calculated as the sum of the coupling between all such sub-segments. ${ }^{20,28-30}$ This approximation retains the simplicity and flexibility of the Förster equation but improves the accuracy of the transfer rate constant with respect to the point dipole approximation by an order of magnitude with moderate computational effort. ${ }^{24}$ It has been shown to work very well for intrachain exciton diffusion on polymeric systems but needs to be tested at smaller chromophore separations than those encountered, on average, in conjugated polymer films. Note that even in this weak limit, the interchromophore couplings modify the excitonic levels and therefore the intrinsic radiative and nonradiative deactivation rates. ${ }^{31}$

In order to explore the applicability of the line-dipole approximation in more closely packed organic semiconductors, we have applied ultrafast spectroscopy on isolated supramolecular nanoassemblies in solution, built by self-assembly of oligo- $p$-phenylenevinylene derivative monofunctionalised with ureido-s-triazine (MOPV; see Figure 1 for the molecular structure). ${ }^{32,33}$ These molecules dimerize by quadruple hydrogen bonding in nonpolar solution. Solvophobic and $\pi-\pi$ interactions result in thermotropically reversible supramolecular assembly, with a relatively sharp transition temperature between assembled and dissolved phases that is dependent on solution concentration but is typically around $55-65^{\circ} \mathrm{C}$ in $10^{-5}-10^{-4} \mathrm{M}$ dodecane solution. Figure 1 shows a schematic representation of these architectures. In MOPV assemblies, supramolecular chirality is induced by chiral aliphatic side groups and adjacent oligmers are offset by a small and regular relative angle. ${ }^{34}$ The nanostructures are a good model system to investigate whether the Förster-like formalism within the line-dipole approximation is sufficient to describe exciton dynamics in closely packed semiconductor polymer systems as the chromophore conformation is undrestood.

In previous publications, we have presented experimental evidence for fast exciton diffusion within MOPV architectures by measuring diffusion-assisted exciton transfer to guest acceptor molecules, ${ }^{35,36}$ nonradiative traps, ${ }^{37}$ and exciton bimolecular annihilation at higher exciton densities. ${ }^{38} \mathrm{Here}$, we model ultrafast experimental data with a model based on RET within the line-dipole approximation in a Monte Carlo scheme. (We model RET events between localized flurophores and chromophores; parallel work based on quantum chemical calculations and a modified master equation explores the role of aggregates in the acceptor distribution. ${ }^{34}$ ) We focus on photoexcitation density regimes where exciton bimolecular annihilation dynamics play a significant role in ultrafast transient data. We have chosen to do so because RET plays a dual role in dictating these dynamics in these OPV systems. First, exciton diffusion within the bulk semiconductor redistributes the timedependent population such that the bimolecular annihilation probability varies with time. Second, in green-emitting materials such as in this OPV supramolecular system, direct, long-range RET interactions between excitons (as opposed to purely diffusion-limited collisional events) are mainly responsible for bimolecular annihilation. ${ }^{39}$ RET and exciton bimolecular annihilation dynamics can therefore be treated with the same microscopic photophysical models. The question that we wish to explore here is can we apply the Förster-like line-dipole scheme to capture the interplay of the fast RET and bimolecular annihilation dynamics in these nanostructures with the same success as in isolated polymer chains in solution? We find that the model is applicable qualitatively, but it fails to reproduce all of the experimental data quantitatively and instead requires a fitting constant (termed a "reduction parameter" here) to increase the predicted transfer rates to represent the experimental measurements. However, we apply the adjusted model to study the role of exciton bimolecular annihilation dynamics in the overall RET processes successfully. We discuss the limitations of the line-dipole approximation in this regime of RET and possible causes of the quantitative failure of the line dipole approximation in the context of MOPV.

\section{Experimental Techniques}

The synthesis of MOPV has been described in detail elsewhere. ${ }^{32,33}$ The material was dissolved in anhydrous dodecane at concentrations around $10^{-4} \mathrm{M}$ and then kept under inert atmosphere except during measurements.

The femtosecond transient absorption apparatus used a homebuilt ultrafast laser system based on the design of Backus et al. ${ }^{40}$ Briefly, $15-f$ s pulses were produced in a mode-locked Ti: sapphire oscillator (KMLabs TS pumped by $4.5 \mathrm{~W}, 532 \mathrm{~nm}$ output from a Spectra-Physics Millennia V laser). The pulse train was amplified using the chirped-pulse-amplification scheme at a repetition rate of $1 \mathrm{kHz}$. This was achieved in a home-built multipass Ti:sapphire amplifier ${ }^{40}$ pumped by the $10-\mathrm{W}, 527-$ $\mathrm{nm}$ output of a Spectra-Physics Evolution X laser. The resulting pulses had a pulse width of $70 \mathrm{fs}$ and a pulse energy of $750 \mu \mathrm{J}$ and were centered at a wavelength of $800 \mathrm{~nm}(1.55 \mathrm{eV}$ photon energy).

The sample was excited with 3.1-eV pump pulses produced by frequency doubling the laser fundamental in a $0.5-\mathrm{mm}$ pathlength $\beta$-BBO crystal. The pump power was varied with calibrated neutral density filters, and the beam was mechanically chopped at $500 \mathrm{~Hz}$. The transient absorption of the sample was 
probed with a single-filament white-light continuum (approximately from 1.2 to $3.0 \mathrm{eV}$ ) produced by focusing a fraction of the fundamental into a sapphire window. Pump pulses were delayed with respect to probe pulses with a computer-controlled optical delay stage. The beams were linearly polarized with a relative angle of $0^{\circ}, 54.7^{\circ}$, or $90^{\circ}$ in parallel, magic-angle, and perpendicular configurations respectively. The probe and the pump beams were focused on the sample cell to $\sim 50$ and 150 $\mu \mathrm{m}$ spots, respectively (with an angle between beams of approximatively $20^{\circ}$ ). The probe beam and a reference beam (not transmitted through the sample) were imaged through a 0.25-m monochromator and detected with a pair of avalanche photodiodes. Pump-induced variations in transmission $(\Delta T / T)$ were calculated using a real-time algorithm which digitized each probe laser shot, applied statistical criteria to reject shots where the integrated intensity varied outside a predetermined deviation from the mean intensity and averaged the data over several thousand shots per time delay point. The instrument response function had a full width at half-maximum of less than $120 \mathrm{fs}$.

We applied time-correlated single photon counting (TCSPC) to measure excited-state lifetimes. The MOPV solution was excited with a pulsed diode laser (PicoQuant LDH400, $20 \mathrm{MHz}$, 70 ps FWHM, $407 \mathrm{~nm}$ ). The photoluminescence was detected with a microchannel plate photomultiplier (Hamamatsu) coupled to a spectrometer and TCSPC electronics (Edinburgh Instruments Lifespec-ps and VTC900 PCI card). The temporal resolution was to $80 \mathrm{ps}$, whereas the spectral resolution was close to $4 \mathrm{~nm}$.

\section{Model}

We apply similar methodology to that in a separate report of intrachain exciton diffusion in a polythiophene derivative. ${ }^{30}$ Each Monte Carlo loop consists of two stages. The algorithm first generates the supramolecular assembly and populates it with excited states, and then carries out a time-loop where each excitation evolves through RET (to unoccupied sites or through bimolecular annihilation) or undergoes intrinsic decay. Typically, we averaged over 1000-5000 Monte Carlo loops (10002000 trajectories) to obtain reasonable statistics.

The assemblies consist of a double helicoidal packing of oligomers with a random, uncorrelated energy distribution. The relative oligomer angle $\left(6^{\circ}\right)$ and separation $(3.5 \AA)$ were deduced from molecular mechanics simulations, ${ }^{34}$ while the assembly radius was measured. ${ }^{33}$ To save computing time, the size of the stack was normally fixed to be 100 oligomers (which implied a length much below the experimental values). This size is justified as the excitons do not explore the entire stack (see below).

The site energy distribution was assumed to be Gaussian with the inhomogeneous broadening as its standard deviation $\sigma_{\text {inh }}$. To determine the homogeneous and inhomogeneous broadening, the absorption, PL, and circular dichroism spectra were fitted with two vibronic progressions of Gaussian functions convoluted with a Gaussian disorder. ${ }^{34}$ The two vibration modes have respectively high frequency $\left(1400 \mathrm{~cm}^{-1}\right)$ and low frequency (80 $\mathrm{cm}^{-1}$ ) to reproduce the bond-length and dihedral deformations associated with exciton formation. The Huang-Rhys factor of the high-frequency mode, $S=1$, was adapted to the vibronic progression of the PL and absorption spectra, whereas the Huang-Rhys factor of the low-frequency mode, $S=15$, was chosen to reproduce the large experimental Stokes shift. The homogeneous and inhomogeneous broadening were found to be 4 and $40 \mathrm{meV}$, respectively. ${ }^{34}$ The RET transfer rate and thus the spectral overlap (see eq 1) should satisfy the detailed balance principle by following a Boltzmann distribution at roomtemperature such that

$$
\frac{I_{i \rightarrow j}\left(E_{i}, E_{j}\right)}{I_{i \leftarrow j}\left(E_{j}, E_{i}\right)}=\exp \left(\frac{-\Delta E}{k T}\right)
$$

where $E_{i}$ and $E_{j}$ denote the energy of two chromophores, $\Delta E=$ $E_{j}-E_{i}$ is the energy difference, $k$ denotes the Boltzmann constant, and $T$ is the temperature. The exciton energy is assumed to be the site energy of the excited oligomer. The probability of exciton generation by the linearly polarized laser field, $P_{i}$, depends on the oligomer orientation with respect to the electric field vector and the ground-state site energy

$$
P_{i}=A_{\mathrm{ex}}\left(\vec{t}_{i} \cdot \vec{z}\right)^{2} \epsilon\left(\hbar \omega-E_{i}\right)
$$

where $\hbar \omega$ is the excitation photon energy and $E_{i}$ is the oligomer energy, $A_{\text {ex }}$ is proportional to the excitation fluence, $\vec{t}_{i}$ is the normalized transition dipole moment orientation vector of the oligomer, $\vec{z}$ is the normalized orientation vector of the excitation polarization, and $\epsilon(E)$ is the homogeneous absorption line shape.

The golden rule describes incoherent energy transfer in the limit of weak coupling between donor $D$ and acceptor $A$ as given by eq $1 . V_{\mathrm{DA}}$, the coulomb integral for transition from donor $D$ to acceptor $A$, is calculated within the line-dipole approximation, using the following expressions:

$$
V_{\mathrm{DA}} \propto \sum_{k, l}^{l d} \Psi_{k}\left|\kappa_{k l}\right| R_{k l}^{-3} \Psi_{l}
$$

$$
\Psi_{k}=\frac{\sin \frac{\pi k}{l_{d}+1}}{\sum_{a} \sin \frac{\pi a}{l_{d}+1}}
$$

In this approach, we partition the conjugated segments into a number $l_{d}$ of sub-segments, denoted as $k$ and $l$ for donor and acceptor, respectively. We choose to describe the center-of-mass excited-state wavefunction $\Psi_{l, k}$ with that corresponding to a point-particle in a finite periodic linear potential as described by Beenken and Pullerits, who derived eq 5.24 This is a reasonable approximation for the wavefunction of the first excitonic state in polymeric semiconductors. The number of subsegments $/_{d}$ was varied over a range spanning 1 and up to 15. In eq $4, \kappa_{k l}$ is a factor accounting for the relative orientation of the subsegments and $R_{k l}$ is the separation between the centers of the two (sub)-dipoles $k$ and $l$. This orientation factor is given by the following relation:

$$
\kappa_{k l}=\left(\vec{t}_{k} \cdot \vec{t}_{l}\right)-3\left(\vec{t}_{k} \cdot \vec{R}_{k l}\right)\left(\vec{t}_{l} \cdot \vec{R}_{k l}\right)
$$

The normalized vectors $\vec{t}_{k, l}$ denote the subsegments $k$ and $l$ and $\vec{R}_{k l}$ is the normalized vector connecting their centers. By replacing $\kappa$ and $R^{-3}$ terms in the usual Förster deviation ${ }^{41}$ with the sum from eq 4 and inserting it into eq 1 , we arrive at the modified line-dipole Förster equation:

$$
k_{\mathrm{RET}}^{\mathrm{LR}}=\frac{1}{\tau_{\mathrm{D}}} \frac{9000 \ln (10) \Phi_{\mathrm{D}}}{128 \pi^{5} N n_{\mathrm{solv}}{ }^{4}} I_{\mathrm{DA}}\left[\sum_{k, l}^{l_{d}} \Psi_{k}\left|\kappa_{k l}\right| R_{k l}{ }^{-3} \Psi_{l}\right]^{2}
$$

Here, $\tau_{\mathrm{D}}$ represents the lifetime of the donor site (taken from the decay of the wavelength-integrated magic-angle PL mea- 
sured with $\left.\mathrm{TCSPC}^{31}\right), \Phi_{\mathrm{D}}$ is the quantum yield of PL of the donor site (taken from previous measurements ${ }^{31}$ ), $N$ is Avogadro's number, and $n_{\text {solv }}$ is the refractive index of the solvent. As described previously, $I_{\mathrm{DA}}$ is the overlap integral of the homogeneously broadened spectra of the chromophores D and A, which depends on the difference in site energies.

The rate of exciton-exciton annihilation (EEA) can be obtained from multiexciton density matrix theory, ${ }^{42}$ however, in this paper, we want to present a simple microscopic incoherent model based on RET rates. Thus, the annihilation process is modeled here as a RET process from one exciton to the other which promotes the acceptor to a higher excitonic state (labeled here as $S_{2}$ ) and the donor to the ground state. The fate of the $S_{2}$ exciton is unclear as it can dissociate into charges or relax to the $S_{1}$ exciton in various timescales. ${ }^{39,43,44}$ For simplicity, we choose to ignore the fate of $S_{2}$ in our simulations, so that each annihilation process simply removes two excitons. (Indeed, using a mesoscopic rate-equation model of bimolecular annihilation dynamics in these nanostructures, we concluded that this is not an important charge generation pathway. ${ }^{38}$ ) The EEA rate should include an overlap integral of the absorption $S_{1} \rightarrow$ $S_{2}$ and the PL spectra, which depends on the difference in site energies. The $S_{1}$ absorption appears in the transient absorption spectrum of MOPV, but as it is difficult to isolate the absorption band from other overlapping transition (see below), we prefer to model this unknown integral with a floating amplitude $A_{\text {EEA }}$ and various functional dependences on the difference in site energies $(f(\Delta E)$, see section IV):

$$
k_{i j}^{\mathrm{EEA}}=A_{\mathrm{EEA}} f(\Delta E)\left[\sum_{k, l}^{l_{d}} \Psi_{k}\left|\kappa_{k l}\right| R_{k l}^{-3} \Psi_{l}\right]^{2}
$$

At each time step, for each exciton, all possibilities of annihilation with any other excitons in the nanostructure are tested, and then all possible RET events to unoccupied sites and the intrinsic decay (with constant probability) are tested in a random order. To simplify the computation, we use the classic point-dipole approximation $\left(l_{d}=1\right)$ when the jump distance exceeds six times the size of the oligomers and neglect it completely when it exceeds 20 times the size of the oligomers. We monitor the random walk of the excitons within the stacks with several ensemble-averaged quantities which are averaged over all Monte Carlo loops. These are the average exciton energy, the rootmean-square displacement, and the exciton anisotropy $r$ defined by

$$
r(t)=\frac{\sum_{i}\left(\vec{t}_{i} \cdot \vec{z}\right)^{2}-\sum_{i}\left(\vec{t}_{i} \cdot \vec{x}\right)^{2}}{\sum_{i}\left(\vec{t}_{i} \cdot \vec{z}\right)^{2}+2 \sum_{i}\left(\vec{t}_{i} \cdot \vec{x}\right)^{2}}
$$

where $\vec{t}_{i}$ denotes the normalized exciton vector, $\vec{z}$ is the direction of excitation polarization, and $\vec{x}$ is a reference vector perpendicular to it.

Note that this model does not take into account the Gaussian geometries of the pump and probe beams. However, by modeling bimolecular annihilation dynamics with a mesoscopic rate equation, we showed in a previous publication that this refinement did not change significantly the results. ${ }^{38}$ Another approximation of this model is the instantaneous creation of excitons which does not reproduce the instrument-limited rise of the exciton signal. This is not strictly correct when considering dynamics on timescales of around one picosecond but is nevertheless used here for simplicity.

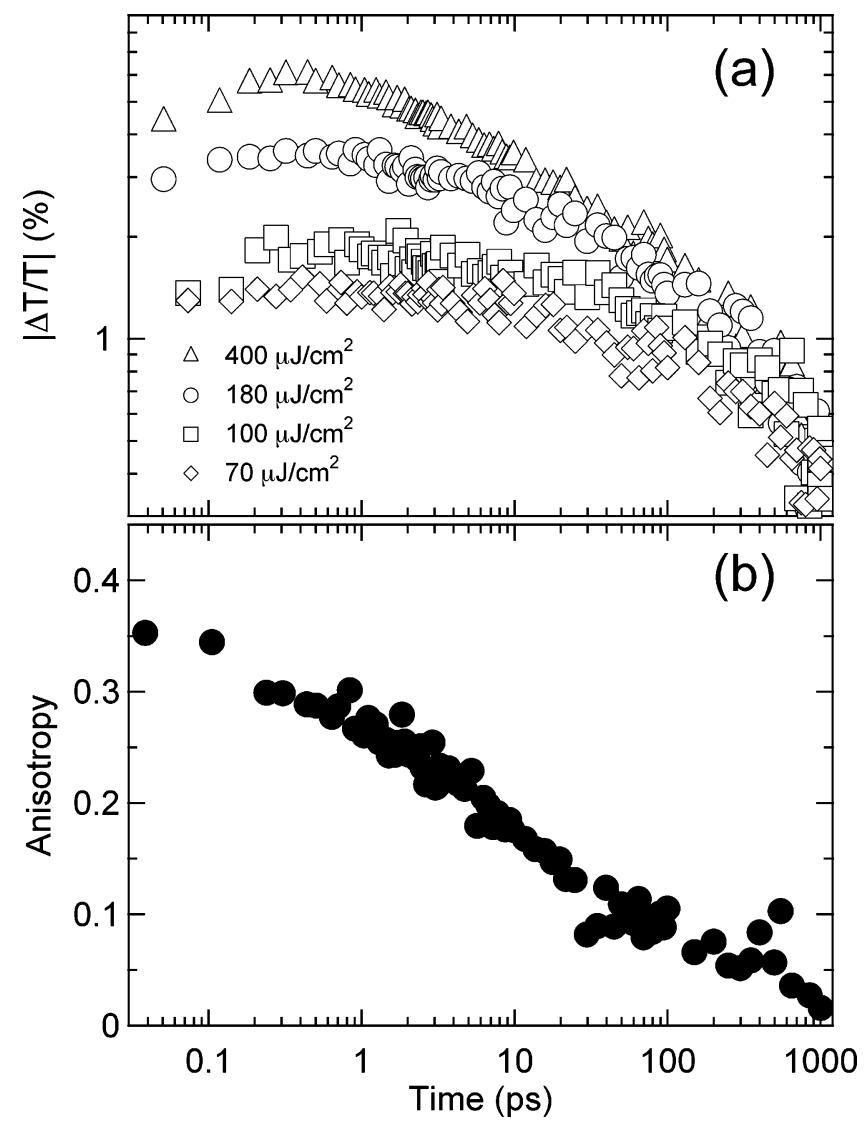

Figure 2. (a) TA signal in the magic angle configuration between the polarization of pump and probe light and at a probe photon energy of $1.38 \mathrm{eV}$ plotted against the pump-probe delay. The various pump fluences are denoted in the inset. (b) Anisotropy signal at the same probe photon energy as above and a fluence of $50 \mu \mathrm{J} / \mathrm{cm}^{2}$, plotted against pump-probe delay. The anisotropy was extracted from absorption transients with parallel and orthogonal relative polarization orientation and eq 10.

\section{Results}

The consequences of supramolecular assembly on MOPV are various, and we have discussed them in a series of papers. ${ }^{31,35-38,45}$ In this paper, we focus on the ultrafast exciton dynamics from around 100 fs to 1000 ps using transient absorption (TA) spectroscopy. Martini et al. have demonstrated that deriving time-resolved exciton population from stimulated-emission TA signal is problematic, especially in regimes where bimolecular annihilation dynamics are significant. ${ }^{46}$ This is due to overlapping spectral contributions from stimulated emission (positive $\Delta T / T)$ and photoinduced absorption from secondary species such as polaron pairs (negative $\Delta T / T$ ), each with different decay kinetics. We therefore choose to monitor exciton population by probing the photoinduced absorption (PA) of the $1 \mathrm{~B}_{u}$ state. ${ }^{38}$ We find that the PA at $\sim 1.4 \mathrm{eV}$ displays a similar decay as the spectrally integrated, time-resolved PL data over long time scales (measured with TCSPC, not shown here) and we will therefore use this transient signal as a probe for exciton population on picosecond timescales.

Figure 2a displays the PA dynamics at $1.38 \mathrm{eV}$ probe photon energy for various excitation fluences. For the two highest pump fluences, there is clearly a pump-dependent ultrafast decay characteristic of EEA. At the two lowest pump fluences, the decay is roughly similar which shows that the decay associated with EEA diminishes at low exciton density, when the average exciton population per stack is lower than one. As we reported previously, ${ }^{38}$ this is characteristic of exciton bimolecular an- 
nihilation processes; the decay of population at early time is dependent on the population density. Part b displays the timedependent anisotropy at low excitation fluence, defined by

$$
r=\frac{\Delta T_{\|}-\Delta T_{\perp}}{\Delta T_{\|}+2 \Delta T_{\perp}}
$$

where $\Delta T_{\|}\left(\Delta T_{\perp}\right)$ is the TA signal with parallel (orthogonal) relative orientation of the polarization of pump and probe laser beams. This quantity has an initial value close to 0.4 , which is expected for a dipole-allowed transition between two nondegenerate levels in a large ensemble of randomly orientated molecules. In the MOPV system in solution studied here, the orientation of the nanostructures is random. After approximately $50 \mathrm{ps}$, the anisotropy reaches a value of 0.1 . This is expected if the memory of the excitation polarization is lost in two dimensions but kept in the third, namely if the exciton transition dipole stays in a plane. This is the case in the columnar structures denoted schematically in Figure 1. This then decays toward zero (corresponding to no memory of the initial orientation distribution) on a nanosecond time scale, probably due to rotational diffusion of the stacks. The rotational diffusion time constant for these naostructures can be estimated using a simple hydrodynamic model and is found to be in the order of 500 ns. ${ }^{47}$ Figure 2 thus gives a strong indication of rapid $(<50 \mathrm{ps})$ exciton diffusion along the stack, in agreement with our previous work applying photoluminescence up-conversion techniques. ${ }^{37}$

In the following subsections, we apply the incoherent hopping model described in section III to explore these dynamics. We first consider the probability of energy transfer to nearest neighbor sites within a Förster model using the point-dipole approximation $\left(l_{d}=1\right)$ in section IV.A. We then re-evaluate these probabilities within the line-dipole approximation $\left(l_{d}>\right.$ 1) in Section IV.B. Finally, we model the femtosecond transient absorption data presented above with the Monte Carlo scheme and evaluate the ability of the point-dipole and line-dipole probabilities to reproduce the ensemble data (section IV.C).

A. Förster Hopping Probabilitites. We first consider the probability that a typical exciton undergoes a single transfer event to its nearest neighbor. We consider the probability for energy transfer from the occupied donor site to the adjacent acceptor site in the same helix (co-facial hops) and to its dimer neighbor, across the hydrogen bonds (lateral hops). (See eq 7 for the definition of these probabilities.) The probabilities with $l_{d}=1$ correspond to the Förster probabilities within the pointdipole approximation. Assuming the supramolecular structure described in section III and the functional form of the centerof-mass excitonic wavefunction (eq 5), the probability of cofacial transfer is computed to be a factor of $\sim 2 \times 10^{5}$ higher than for lateral transfer (see Figure 3 ). This ratio drops to $\sim 3$ $\times 10^{3}$ for transfer two sites away within and across the helix, to $\sim 20$ for four sites away in either helix, and to $\sim 3$ for acceptors located ten sites away. Furthermore, this model predicts that the Förster probability of energy transfer to an unoccupied site that is six molecules away in the same helix is comparable to the probability of the nearest-neighbor lateral transfer.

B. Evolution of Hopping Probabilities with /d. Figure 4 shows the evolution of the probability for cofacial jumps (open triangles) and across the hydrogen-bonds (filled circles) with the number of sub-segments in the line dipole approximation $(/ d)$. Upon increasing $/ d$, the probability of nearest cofacial jump decreases, and that of a lateral jump increases. The ratio of probabilities of cofacial and lateral transfer events for $l_{d}=4$ is
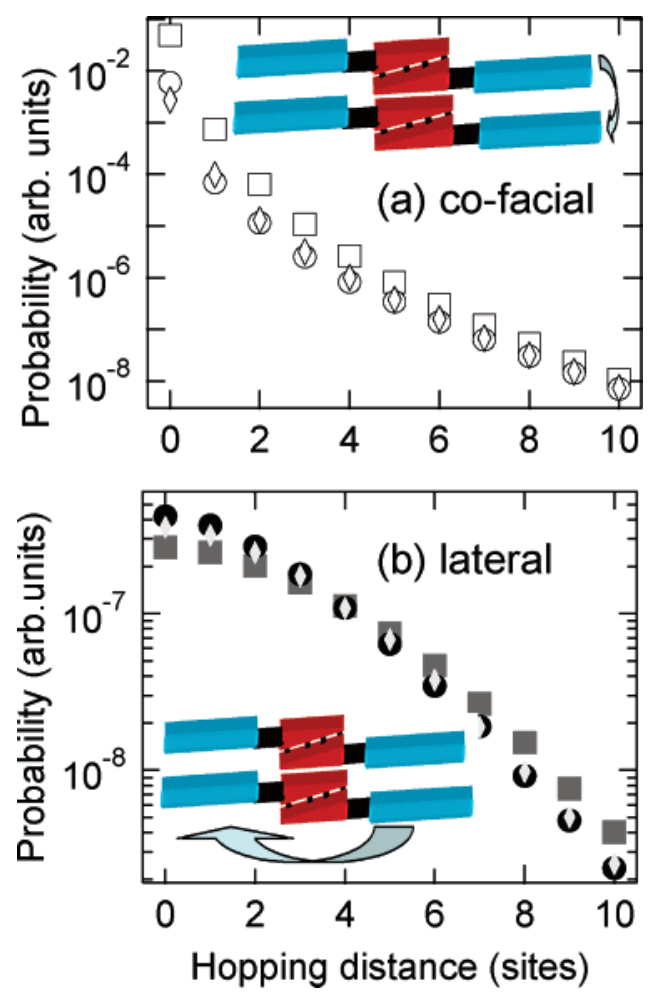

Figure 3. Normalized transfer rates for hops on the same chiral side (open symbols, part a) and hops across the hydrogen-bonding groups (filled symbols, part b) plotted against the hopping distance in units of oligomers, for $l_{d}=1$ (squares), 4 (circles), and 15 (diamonds).

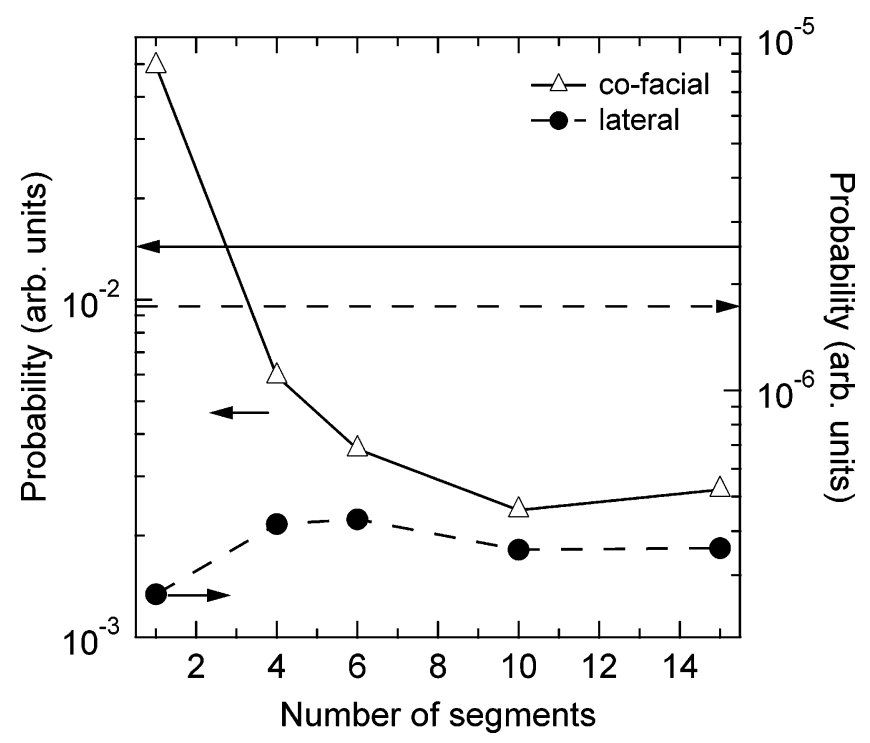

Figure 4. Transfer probabilities for nearest-neighbor hops (open triangles) and lateral hops across the hydrogen bonds (filled circles) plotted against number of segments used in the line dipole approximation $(/ d)$. The horizontal lines are the transfer rates that provide the best fit to the experimental data; the continuous line corresponding to the left-axis scale is for co-facial transfer and the dashed line corresponding to the right-axis scale is for lateral transfer.

$\sim 1.4 \times 10^{4}$, roughly a factor of 20 smaller than for $l_{d}=1$. With respect to the line-dipole approximation, the point-dipole approximation therefore overestimates the probability of a jump to its nearest neighbor but underestimates the probability of a lateral jump across the hydrogen bonds. This trend is in agreement with our related simulations of intramolecular exciton transport in a polythiophene derivative, where transfer in cofacial segments in a chain adopting coiled conformations was under- 
estimated by the point-dipole approximation, but those for adjacent sites along the chain were overestimated. ${ }^{30}$ Note that the probabilities for cofacial transfer seem to converge when the number of subsegments approaches $\sim 8$, indicating that the convergence of the line-dipole approximation is already achieved with 8 segments. We now return to Figure 3, which also displays the evolution of the hopping probability with the hopping distance for $l_{d}=4$ and 15 . The largest difference in the hopping probability is seen between $l_{d}=1$ and 4 for the case of cofacial hops over distances of $1-4$ sites. This emphasizes the failure of the point-dipole approximation over distances where the spatial extent of the excited-state wavefunction needs to be considered. ${ }^{21,34}$ Significantly, the dependence on the lateral distance has already converged with 4 segments due to the larger donor-acceptor separation across the hydrogen bond.

C. Comparison between Model and Experiment. We now turn to the comparison of the results of the model with the experimental data in Figure 5. Part a compares Monte Carlo simulations and the experimental exciton population density, determined by $N=(-\sigma z)^{-1} \ln (\Delta T / T+1)$, with $\sigma$ the excitedstate absorption cross section and $z$ the laser penetration depth. Part b compares the experimental PL average energy, and part c compares the experimental polarization anisotropy. The continuous lines are the best fits and the other lines are variations in the model parameters. The average exciton density on the stacks was varied using $A_{\text {ex }}$ (eq 3 ) in the probability of exciton creation. In a previous paper, ${ }^{38}$ we showed that the peak exciton density was around $4 \times 10^{15} \mathrm{~cm}^{-3}$ at a pump fluence close to $600 \mu \mathrm{J} / \mathrm{cm}^{2}$. This allows us to get the experimental exciton densities on the stacks (approximately 0.5 and 5 per 100 oligomers at the two experimental fluences used in Figure 5) and to determine $A_{\mathrm{ex}}$ required at these fluences.

We seek to test the line-dipole approximation to model energy transport in this class of supramolecular nanostructures, and so all of the parameters used in the model were determined independently; the only parameter that was varied was $l_{d}$ as was described above, and we used the converged value of $l_{d}=6$ in the model of the ultrafast decay kinetics. In order to obtain the best modeled decay kinetics, we had to introduce a parameter $R_{e}$ multiplying the converged transfer rates in Figure 4 to speed up the dynamics. The horizontal lines in Figure 4 are the probabilities needed to reproduce the experimental data. The coefficient $R_{e}$ that modifies the transfer rates allows us to keep the same dependence on distance, orientation, and energy difference while increasing the overall probabilities. The need for such a parameter cannot be explained by experimental errors or lack of convergence of the Monte Carlo simulation. Note that this parameter was not needed in our previous simulations of intramolecular energy migration in a conjugated polymer, where the same type of simulation with independently measured quantities reproduced the ultrafast data with the converged value of $l_{d} \cdot 30$

To illustrate the impact of the reduction parameter $R_{e}$, we used the point-dipole approximation $\left(l_{d}=1\right)$ to extract a Förster radius from our transfer rates (for this we neglect the different dependences in the interchromophore separation). The pointdipole approximation predicts a value of around $1.4 \mathrm{~nm}$ for an isoenergetic hop (four times the interchromophore separation), but to reproduce the experimental data, values between 1.6 and $2.1 \mathrm{~nm}$ have to be used (around six times the inter-chromophore separation). The main parameters extracted from this model are summarized in Table 1.

Applying the reduction parameter reported in Table 1, we now consider the parameters in the model that determine the

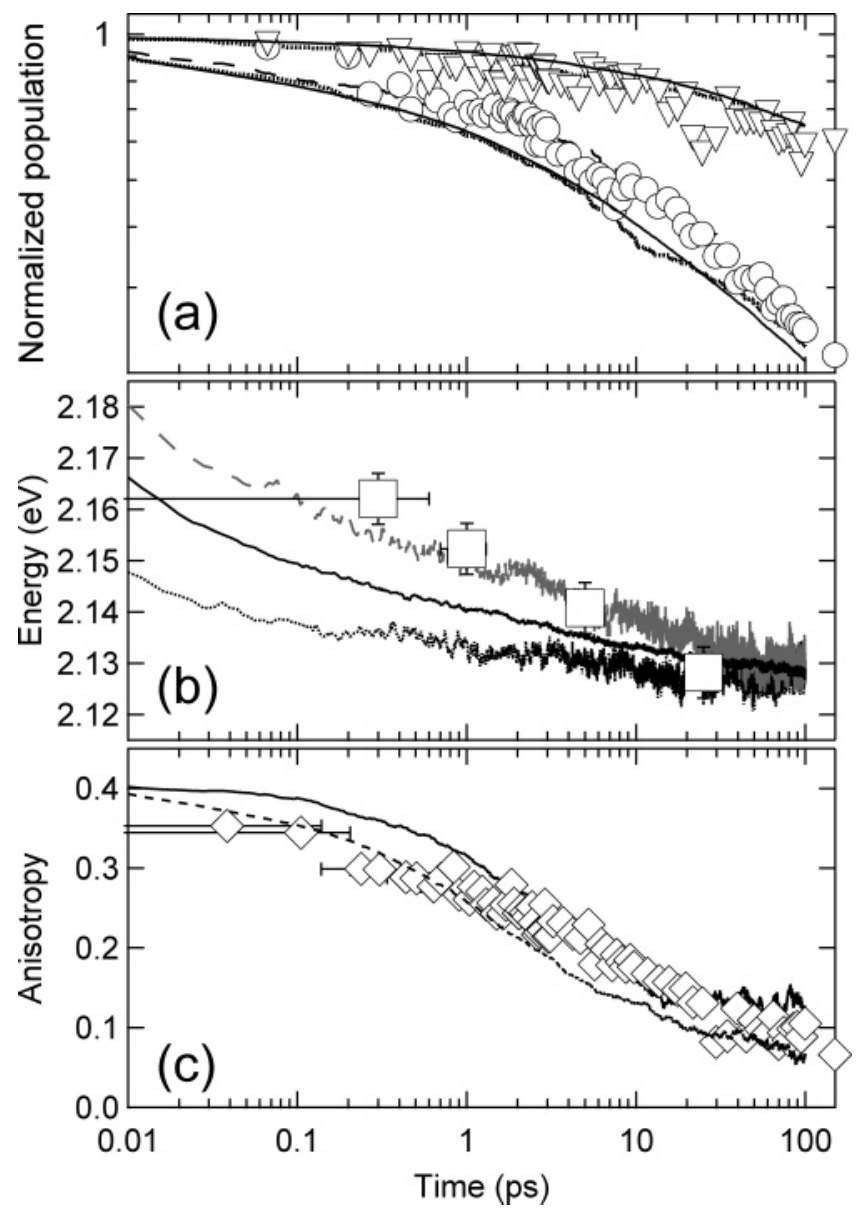

Figure 5. (a) Normalized exciton population density deduced from FTA signal at $1.38 \mathrm{eV}$ and pump fluences of $1 \mathrm{~mJ} / \mathrm{cm}^{2}$ (open circles) and $130 \mu \mathrm{J} / \mathrm{cm}^{2}$ (open inverted triangles). The peak exciton density at the lower fluence is deduced to be $6 \times 10^{14} \mathrm{~cm}^{-3}$ and that at the higher fluence is $5 \times 10^{15} \mathrm{~cm}^{-3}$. Also shown are various outputs of the simulation. The best fits are represented by thick solid lines, simulations with high EEA rate are represented by dotted lines and those with low EEA rates with dashed lines. (b) Average PL energy deduced from photoluminescence up-conversion spectra ${ }^{37}$ at very low excitation fluences (open squares). The best fit from the simulation is displayed as a solid line, the low standard deviation of energy site distribution as a dotted line, and the high standard deviation of energy site distribution as a gray dashed line. (c) Exciton anisotropy extracted from FTA data with parallel and perpendicular relative pump and probe polarization orientations at a probe photon energy of $1.38 \mathrm{eV}$ and a fluence of 50 $\mu \mathrm{J} / \mathrm{cm}^{2}$ (diamonds) and simulation output at high (dashed line) and low exciton (solid line) densities.

TABLE 1: Parameters Used in the Model to Produce the Best Fits to the Data in Figure 5

$\begin{array}{ll}\text { number of oligomers } & 100 \\ \text { separation of oligomers } & 3.5 \AA \\ \text { relative angle } & 6^{\circ} \\ \text { stack radius } & 3 \mathrm{~nm} \\ \text { energy distribution }\left(\sigma_{\mathrm{inh}}\right) & (35 \pm 10) \mathrm{meV} \\ \text { number of dipole segments }\left(l_{d}\right) & 6 \\ \text { reduction parameter }\left(R_{\mathrm{e}}\right) & 4 \pm 1\end{array}$

bimolecular annihilation dynamics. In Figure 5a, we report the effect of the annihilation rate (by varying $A_{\mathrm{EEA}}$ in eq 8 ) on the exciton dynamics. In the upper panel, two sets of curves correspond to an EEA rate twice as big as the best fit (dotted lines) and four times smaller (dashed lines). The effect is modest because in the Förster formalism, the effective annihilation radius follows the sixth-root of the rate. If we use the pointdipole approximation, our best-fit value corresponds to a Förster 
radius of $2.3 \pm 0.5 \mathrm{~nm}$ for an isoenergetic hop (7 times the inter-chromophore separation).

We used three functional dependences in the exciton energy difference $(f(\Delta E)$ in eq 8$)$. These were a sum of exponentials, a Gaussian, and a flat line. From the detailed balance principle, the dependence in site energy difference of the transfer rate is exponential (a Boltzmann factor). As the EEA is treated as a RET event, we normally used a sum of two exponentials, $\exp (+\Delta E / k T)+\exp (-\Delta E / k T)$. If the EEA process has a more resonant character, one might expect a higher probability if the exciton energy difference is small and the functional form of $f(\Delta E)$ would be closer to a Gaussian. The simulations show that, in our case, this functional dependence does not influence significantly the ensemble averaged exciton decay.

We turn now to the dependence on the standard deviation of the Gaussian site energy distribution. In Figure 5b, two curves correspond to a standard deviation of the site energy distribution of $10 \mathrm{meV}$ bigger and smaller (dashed and dotted lines, respectively) than the best fit reported in Table 1 (solid line). The effect of this parameter on the dynamic red-shift magnitude is important and allows us to define upper and lower limits on this parameter. Note that the best fit value corresponds roughly to the inhomogeneous broadening predicted by the fitting of the PL, absorption, and circular dichroism spectra. Also, the decay of the average energy does not depend on the exciton density. The two output curves corresponding to the two exciton densities used in the upper panel overlap perfectly (not shown here).

We studied the effect of correlation in site energies. The disorder in site energies can have several origins such as variations in the local packing, in the oligomer internal conformation, or in the surrounding solvent bath. As one might expect correlation in energy from one molecule to the next, ${ }^{25}$ we carried out simulations with medium correlation where the energy of the $n$th oligomer is half the energy of the $(n-1)$ th and half a random number from a Gaussian distribution. This has a strong influence on the dynamic red-shift, and to reproduce the experimental data, the standard deviation of the Gaussian distribution has to be increased by a factor of 2 to less physical values. We therefore elected to exclude it from the simulations yielding parameters reported in Table 1, but note that a full treatment of spatial disorder correlation requires a quantum mechanical treatment as performed by Spano et al., who use a Holstein-like Hamiltonian to describe optical excitations in MOPV nanostructures. ${ }^{48}$

The preparation of the system with the exciton creation probability from eq 3 is not unique and different pump pulse conditions might change the selectivity of the pump. However, we tried preparing the system with no energy dependence (without the absorption term in eq 3), and it does not change significantly the exciton dynamics. This is probably due to the relatively narrow distribution of site energies compared to the homogeneously broadened absorption spectrum.

Figure $5 \mathrm{c}$ demonstrates that the decay of the exciton anisotropy depends on the exciton density used in the simulation. At high exciton densities, when exciton bimolecular annihilation is dominant, the anisotropy decays faster than at low exciton densities. This effect is related to the angular dependence of the exciton creation probability. If an oligomer is almost parallel to the excitation polarization, the probability of creating an exciton on this oligomer is very high. As the relative oligomer angle is very small, the neighboring oligomers will have a high probability of exciton creation as well. In this case, a small group of oligomers could contain several excitons that will annihilate

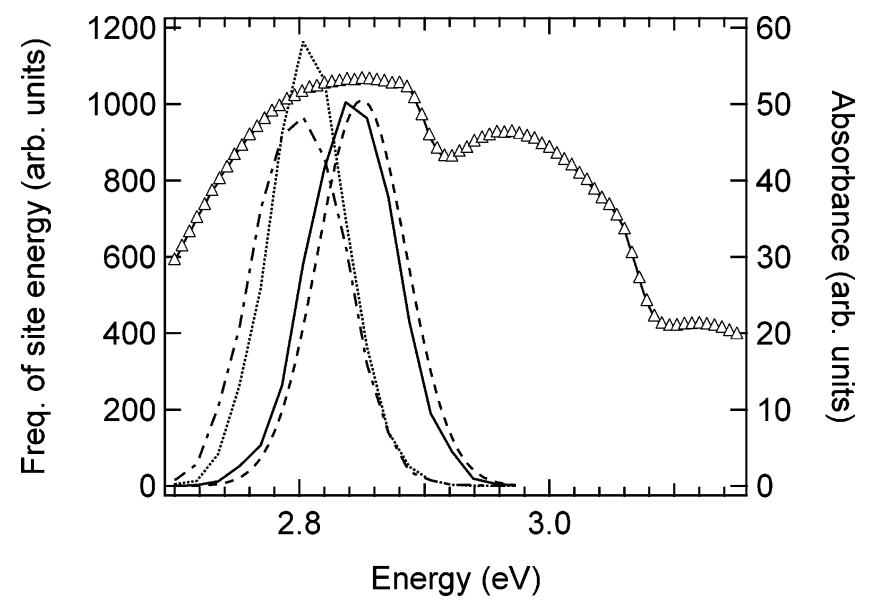

Figure 6. Initial distribution of site energies (dashed line, left axis) and homogeneously broadened absorption spectrum (solid line and open triangles, right axis) extracted from the Monte Carlo simularion. Also shown is the exciton population density plotted against site energy at $4 \mathrm{fs}$ (solid line), 1 ps (dotted line) and 100 ps (dotted-dashed line) after excitation (left axis).

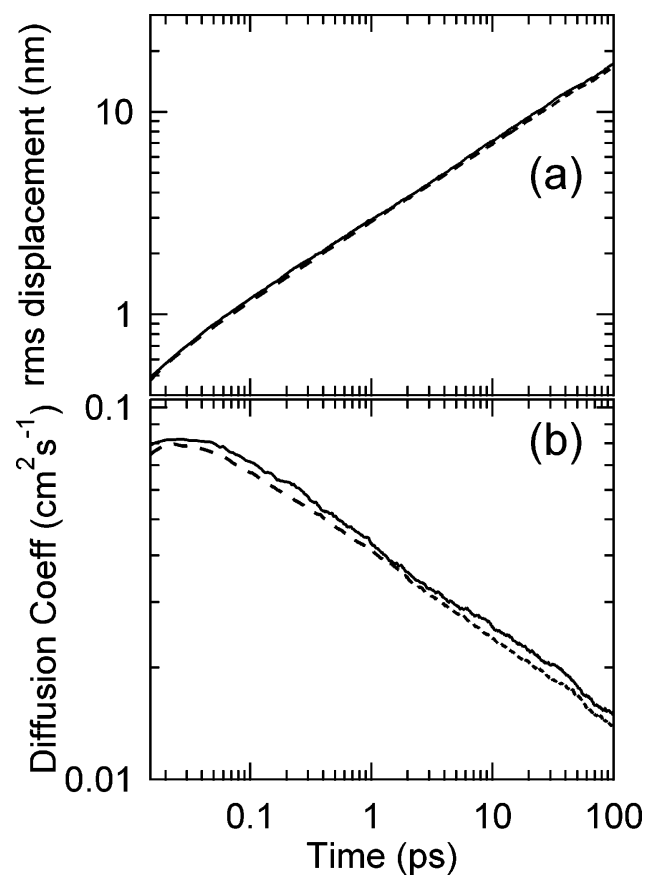

Figure 7. (a) Normalized root-mean-square exciton displacement and (b) exciton diffusion coefficient obtained from the simulation plotted against time. The solid line is extracted from a simulation at low pump fluence and the dashed line is extracted from a simulation at high pump fluence. Here "high" and "low" fluence correspond to the range used in Figure 5.

very quickly. The decay of these excitons will also decrease the anisotropy as they are well aligned with the initial excitation polarization. Therefore, exciton annihilation is expected to enhance the decay of the exciton anisotropy. Our experimental anisotropies were all taken at a low excitation fluence and are reproduced satisfactorily by our simulation.

With the results of our model, we can extract microscopic information that is not directly available otherwise. Figure 6 plots the distribution in exciton site energy at various times. Figure 7 (part b) shows the evolution of the exciton diffusion coefficient $D$ with time. This is obtained from the root-meansquare exciton displacement (part a), assuming a dimentionality of one, ${ }^{37}$ by $(2 D t)^{1 / 2}=\sqrt{ }\left\langle x^{2}\right\rangle$. D decreases from a high initial value of $8 \times 10^{-2}$ to $4 \times 10^{-2} \mathrm{~cm}^{2} \mathrm{~s}^{-1}$ in $2 \mathrm{ps}$ and almost to 
$1 \times 10^{-2} \mathrm{~cm}^{2} \mathrm{~s}^{-1}$ in $100 \mathrm{ps}$, a high but still common value in organic semiconductors. ${ }^{49}$

This high initial diffusion rate constant translates into an average hopping time of $80 \mathrm{fs}$. This is approximately twice as fast as that found in the peripheral plant light-harvesting complex LHCII ${ }^{50,51}$ but is, for instance, comparable to RET time constants found in systems involving carotenoids. ${ }^{52}$ This high coefficient is the direct consequence of the chromophore close packing and is necessary to reproduce, for instance, the ultrafast decay of the exciton anisotropy. We note that the values of $D$ extracted here indicate that the exciton does not migrate anywhere near the length of the stacks that we have adopted for this simulation.

Note that the annihilation process has a fixed rate for any given donor-acceptor separation and its importance in the photophysics decreases at low excitation fluences only as the exciton population decreases. With the lowest excitation fluence investigated here, around $10 \%$ of excitons decay through annihilation. This is due to a combination of the high exciton diffusion rate, a significant annihilation radius, and the variation in the numbers of excitons per stack. The latter is important. As the stacks are randomly oriented, some are in a more favorable orientation for exciton photogeneration, so that the number of excitons per stack of 100 oligomers varies from zero ( $70 \%$ of the stacks) to one ( $25 \%$ of them) to four $(1 / 1000$ of them), even when the average number of excitons per stack is less than 0.4 .

\section{Discussion}

The excited-state diffusion model presented here is based on incoherent energy transfer. The golden rule expressed in eq 1 is only valid when coherent interactions can be neglected. Our findings presented in Figure 5 are consistent with the random walk picture that has been used previously by many authors to describe successfully exciton migration in polymer films and solutions. ${ }^{18-20,25,28-30}$ This incoherent process plays an important role in the bimolecular annihilation dynamics modeled here. However, the transfer rates obtained from a direct application of the line-dipole formalism are too low to reproduce the experimental data, even when the method has converged (see Figure 4). Therefore, these discrepancies are not related to the limited convergence of the multipole expansion but more fundamentally to the excited-state description implemented here in the MOPV supramolecular assemblies.

We now discuss the possibilities for the quantitative failure of the line-dipole formalism as applied to MOPV here. First, in MOPV stacks, the interchromophore separation is smaller than what is common in polymeric semiconductors, so the interchromophore couplings might be comparable to the exciton-phonon couplings. In such a case, the exciton diffusion might be coherent on some ultrafast timescales, which would prevent the use of the golden rule (and the line-dipole approximation). We do not think that this is the case on timescales following a few hundred femtoseconds because of the success of this completely incoherent model in reproducing the exciton dynamics after correction with $R_{\mathrm{e}}$ (but we do note, however, that the experimentally measured degree of circular polarization in absorbance and photoluminescence is comparable in the region of spectral overlap between these two spectra ${ }^{48}$ ). This view is in agreement with quantum-chemical simulations of MOPV structures based on a master equation scheme. ${ }^{34}$ Another intriguing possibility not represented in this model is the effect of coherence, not in the donor fluorophore but in the acceptor chromophore. This situation is explored in a parallel publication employing a modified quantum master equation formalism based on incoherent hopping from a localized oligomer to a chromophore comprising aggregates of several oligomers. ${ }^{34}$ That work is based on a significantly more complete quantumchemical description of excitons in MOPV supramolecular structures and demonstrates the importance of aggregation effects in this system. In this scenario, the functional form of the acceptor wavefunction $\Psi_{l}$ (eq 5) used here is a severe misrepresentation, which would lead to an incorrect prediction for the transfer probabilities. Note that this limitation is not a failure of the line dipole formalism itself but of the chosen functional form of $\Psi_{l}$.

Second, even neglecting the point about possible aggregation in the acceptor distribution, the wavefunction $\Psi_{l}$ describing the weight of each sub-dipole might still not be an adequate representation in the case of closely packed oligomers as in MOPV. It can be obtained rigorously from a full quantummechanical calculation of the electronic transition density ${ }^{21-23}$ but is approximated here with the wavefunction of a pointparticle in a finite periodic linear chain (see eq 5). ${ }^{24}$ This approximation might not be valid in our case as the excitonic wavefunctions are distorted by the close packing and, as it is two-dimensional, it fails to reflect the three-dimensional extension. Also, the exciton is a quasi-particle of a bound state of two quasi-particles (the electron and the hole). As such, it has a nonzero extension and any representation as a delocalized point-particle misses the exciton size and the wavefunction describing the internal separation of the electron and the hole. This separation was recently estimated by one of us to be $3 \AA$ for a conjugated segment of PPV of $3 \mathrm{~nm} .{ }^{53}$ Such a value leads to interactions between delocalized dipoles which are not equivalent to interactions between delocalized point-dipoles.

Third, small interchromophore separations could lead to a non-negligible wavefunction overlap in adjacent molecules and change the interchromophore couplings by the addition of an exchange energy term. As the supramolecular assemblies are weakly coupled $\mathrm{H}$-aggregates, ${ }^{54}$ including the exchange interaction would increase the coupling since the exciton wavefunctions would have an antibonding character.

In the outset of this paper, we identified MOPV supramolecular nanostructures as model systems to investigate exciton diffusion dynamics in polymeric semiconductors, including effects of exciton bimolecular annihilation. All of the limitations discussed above do not rule out the use of a scheme based on the line-dipole approximation for that purpose as the model is still very powerful to extract microscopic information from experimental data, even in this so-called intermediate interchromophore coupling regime, which is on the high limit of the average interchromophore coupling in random conjugated polymer films. The quantitative failure of the line-dipole model offers a very interesting window on the physics of chromophores at very close separation. The importance of aggregation effects in the acceptor distribution and the exchange energy should be understood in order to model the physics of closely packed $\pi$-conjugated molecules in the condensed phase. ${ }^{34}$

\section{Conclusion}

MOPV supramolecular assemblies display very fast energy diffusion over early times following photoexcitation with femtosecond pulses. The rates for this are comparable to or faster than those observed in polymeric semiconductors. This is related to the very close packing of the oligomers and the resulting importance of high interchromophore coupling. This also results in a high bimolecular annihilation rate constant compared to disordered conjugated polymer films. 
Using a Monte Carlo scheme, we determined that energy diffusion and bimolecular annihilation processes can be described with the same microscopic model based on a Försterlike model. These two processes, which are both important in the decay of measured polarization anisotropy, are interconnected via the same underlying physics.

Acknowledgment. We are deeply indebted to Freek Hoeben, Pascal Jonkheijm, Albert Schenning, and Bert Meijer at the Eindhoven Institute of Technology for providing materials and for the ongoing, exciting collaboration. We also thank Peter Bobbert and Stefan Meskers for valuable discussions. The work in Cambridge is supported by the UK Engineering and Physical Sciences Research Council (EPSRC) and the Interdisciplinary Research Center for Nanotechnology (Cambridge, UCL, Bristol). C.S. acknowledges the EPSRC for further support within the Advanced Research Fellowship scheme in Cambridge, and the National Science and Engineering Research Council (NSERC) and Canada Research Chairs program for the work in Montréal. C.D. thanks the Cambridge European Trust for partial support within the Isaac Newton Scholarship scheme. The work in Mons is partly supported by the Belgian Federal Government "InterUniversity Attraction Pole in Supramolecular Chemistry and Catalysis (PAI 4/11)" and the Belgian National Fund for Scientific Research (FNRS-FRFC). D.B. acknowledges the FNRS for further support as Research Associate. The CambridgeEindhoven-Mons collaboration was supported by the European Commission (LAMINATE).

\section{References and Notes}

(1) Friend, R. H.; Gymer, R. W.; Holmes, A. B.; Burroughes, J. H.; Marks, R. N.; Taliani, C.; Bradley, D. D. C.; DosSantos, D. A.; Brédas, J. L.; Lögdlund, M.; Salaneck, W. R. Nature (London) 1999, 397, 121.

(2) Cao, Y.; Parker, I. D.; Yu, G.; Zhang, C.; Heeger, A. J. Nature (London) 1999, 397, 414.

(3) Morteani, A. C.; Dhoot, A. S.; Kim, J. S.; Silva, C.; Greenham, N. C.; Murphy, C.; Moons, E.; Cina, S.; Burroughes, J. H. Friend, R. H. Adv. Mater. 2003, 15.

(4) Morteani, A. C.; Ho, P. K. H.; Friend, R. H.; Silva, C. Appl. Phys. Lett. 2005, 86, 163501.

(5) Halls, J. J. M.; Walsh, C. A.; Greenham, N. C.; Marseglia, E. A.; Friend, R. H.; Moratti, S. C.; Holmes, A. B. Nature (London) 1995, 376, 498.

(6) Yu, G.; Cao, J.; Hummelen, J. C.; Wudl, F.; Heeger, A. J. Science 1995, 270, 1789.

(7) Halls, J. J. M.; Arias, A. C.; MacKenzie, J. D.; Wu, W.; Inbasekaran, M.; Woo, E.; Friend, R. H. Adv. Mater. 2000, 12, 498.

(8) Brabec, C. J.; Sariciftci, N. S.; Hummelen, J. C. Adv. Funct. Mater. 2001, 11, 15 .

(9) Snaith, H. J.; Arias, A. C.; Morteani, A. C.; Silva, C.; Friend, R. H. Nano Lett. 2002, 2, 1353.

(10) van Duren, J. K. J.; Yang, X. N.; Loos, J.; Bulle-Lieuwma, C. W. T.; Sieval, A. B.; Hummelen, J. C.; Janssen, R. A. J. Adv. Funct. Mater. 2004, 14,425

(11) Scholes, G. D.; Rumbles, G. Nat. Mater. 2006, 5, 683.

(12) Brabec, C. J.; Winder, C.; Sariciftci, N. S.; Hummelen, J. C.; Dhanabalan, A.; van Hal, P. A.; Janssen, R. A. J. Adv. Funct. Mater. 2002, 12,709 . 54

(13) Greenham, N. C.; Peng, X. G.; Alivisatos, A. P. Phys. Rev. B 1996,

(14) Sun, B.; Marx, E.; Greenham, N. C. Nano Lett. 2003, 3, 961.

(15) Morteani, A. C.; Sreearunothai, P.; Herz, L. M.; Friend, R. H.; Silva, C. Phys. Rev. Lett. 2004, 92, 247402.

(16) Morteani, A. C.; Friend, R. H.; Silva, C. J. Chem. Phys. 2005, 122, 244906.

(17) Morteani, A. C.; Friend, R. H.; Silva, C. In Organic Light Emitting Devices. Synthesis, properties and Applications; Müllen, K., Scherf, U., Eds.; Wiley-VCH: Weinheim, Germany, 2006; pp 36-94.

(18) Scheidler, M.; Lemmer, U.; Kersting, R.; Karg, S.; Riess, W.; Cleve, B.; Mahrt, R. F.; Kurz, H.; Bässler, H.; Göbel, E. O.; Thomas, P. Phys. Rev. B 1996, 54, 5536.

(19) Meskers, S. C. J.; Hubner, J.; Oestreich, M.; Bässler, H. J. Phys. Chem. B 2001, 105, 9139.
(20) Grage, M. M. L.; Pullerits, T.; Ruseckas, A.; Theander, M.; Inganas, O.; Sundström, V. Chem. Phys. Lett. 2001, 339, 96.

(21) Beljonne, D.; Pourtois, G.; Silva, C.; Hennebicq, E.; Herz, L. M.; Friend, R. H.; Scholes, G. D.; Setayesh, S.; Müllen, K.; Brédas, J. L. Proc. Nat. Acad. Sci. U.S.A. 2002, 99, 10982.

(22) Hennebicq, E.; Pourtois, G.; Scholes, G. D.; Herz, L. M.; Russell, D. M.; Silva, C.; Setayesh, S.; Grimsdale, A. C.; Müllen, K.; Brédas, J.-L.; Beljonne, D. J. Am. Chem. Soc. 2005, 127, 4744-4762.

(23) Poulsen, L.; Jazdzyk, M.; Communal, J.-E.; Sancho-Garca, J. C.; Mura, A.; Bongiovanni, G.; Beljonne, D.; Cornil, J.; Hanack, M.; Egelhaaf, H.-J.; Gierschner, J. J. Am. Chem. Soc. in press.

(24) Beenken, W. J. D.; Pullerits, T. J. Chem. Phys. 2004, 120, 2490

(25) Abramavicius, D.; Valkunas, L.; van Grondelle, R. Phys. Chem. Chem. Phys. 2004, 6, 3097.

(26) Herz, L. M.; Silva, C.; Grimsdale, A. C.; Müllen, K.; Phillips, R. T. Phys. Rev. B 2004, 70, 165207.

(27) Beljonne, D.; Cornil, J.; Silbey, R.; Millié, P.; Brédas, J. L. J. Chem. Phys. 2000, 112, 4749.

(28) Grage, M. M. L.; Wood, P. W.; Ruseckas, A.; Pullerits, T.; Mitchell, W.; Burn, P. L.; Samuel, I. D. W.; Sundström, V. J. Chem. Phys. 2003 118,7644 .

(29) Grage, M. M. L.; Zaushitsyn, Y.; Yartsev, A.; Chachisvilis, M.; Sundström, V.; Pullerits, T. Phys. Rev. B 2003, 67, 205207.

(30) Westenhoff, S.; Daniel, C.; Friend, R. H.; Silva, C.; Sundström, V.; Yartsev, A. J. Chem. Phys. 2005, 122, 094903. Westenhoff, S.; Beenken, W. J. D.; Friend, R. H.; Greenham, N. C.; Yartsev, A.; Sundstrom, V. Phys. Rev. Lett. 2006, 97, 166804.

(31) Daniel, C.; Makereel, F.; Herz, L. M.; Hoeben, F. J. M.; Jonkheijm, P.; Schenning, A. P. H. J.; Meijer, E. W.; Silva, C. J. Chem. Phys. 2005, $123,084902$.

(32) Schenning, A. P. H. J.; Jonkheijm, P.; Peeters, E.; Meijer, E. W. J. Am. Chem. Soc. 2001, 123, 409.

(33) Jonkheijm, P.; Hoeben, F. J. M.; Kleppinger, R.; van Herrikhuyzen, J.; Schenning, A. P. H. J.; Meijer, E. W. J. Am. Chem. Soc. 2003, 125, 15941.

(34) Beljonne, D.; Hennebicq, E.; Daniel, C.; Herz, L. M.; Silva, C.; Scholes, G. D.; Hoeben, F. J. M.; Jonkheijm, P.; Schenning, A. P. H. J.; Meskers, S. C. J.; Phillips, R. T.; Friend, R. H.; Meijer, E. W. J. Phys. Chem. B 2005, 109, $10594-10604$.

(35) Hoeben, F. J. M.; Herz, L. M.; Daniel, C.; Jonkheijm, P.; Schenning, A. P. H. J.; Silva, C.; Meskers, S. C. J.; Beljonne, D.; Phillips, R. T.; Friend, R. H.; Meijer, E. W. Angew. Chem. 2004, 43, 1976.

(36) Chang, M. H.; Hoeben, F. J. M.; Jonkheijm, P.; Schenning, A. P. H. J.; Meijer, E. W.; Silva, C.; Herz, L. M. Chem. Phys. Lett. 2006, 418, 196.

(37) Herz, L. M.; Daniel, C.; Silva, C.; Hoeben, F. J. M.; Schenning, A. P. H. J.; Meijer, E. W.; Friend, R. H.; Phillips, R. T. Phys. Rev. B 2003, $68,045203$.

(38) Daniel, C.; Herz, L. M.; Silva, C.; Hoeben, F. J. M.; Schenning, A. P. H. J.; Meijer, E. W. Phys. Rev. B 2003, 68, 235212.

(39) Stevens, M. A.; Silva, C.; Russell, D. M.; Friend, R. H. Phys. Rev. B 2001, 63, 165213.

(40) Backus, S.; Bartels, R.; Thompson, S.; Dollinger, R.; Kapteyn, H. C.; Murnane, M. M. Opt. Lett. 2001, 26, 465.

(41) Scholes, G. D. Annu. Rev. Phys. Chem. 2003, 54, 57

(42) Brüggemann, B.; Herek, J. L.; Sundström, V.; Pullerits, T.; May, V. J. Phys. Chem B 2001, 105, 11391.

(43) Silva, C.; Russell, D. M.; Dhoot, A. S.; Russell, D. M.; Stevens, M. A.; Arias, A. C.; MacKenzie, J. D.; Greenham, N. C.; Friend, R. H.; Setayesh, S.; Müllen, K. Phys. Rev. B 2001, 64, 125211.

(44) Silva, C.; Dhoot, A. S.; Russell, Herz, L. M.; Daniel, C.; Greenham, N. C.; Arias, A. C.; Setayesh, S.; Müllen, K.; Friend, R. H. J. Phys. Condensed Matter 2002, 14, 9803.

(45) Daniel, C.; Herz, L. M.; Silva, C.; Hoeben, F. J. M.; Schenning, A. P. H. J.; Meijer, E. W. Synth. Met. 2004, 147, 29.

(46) Martini, I. B.; Smith, A. D.; Schwartz, B. J. Phys. Rev. B 2004 69, 035204

(47) Westenhoff, S.; Abrusci, A.; Feast, W. J.; Henze, O.; Kilbinger, A. F. M.; Schenning, A. P. H. J.; Silva, C. Adv. Mater. 2006, 18, 1281 (48) Spano, F.; Meskers, C. J.; Hennebicq, E.; Beljonne, D. J. Am. Chem. Soc. 2007, 129, 7044 .

(49) Powell, R. C.; Soos, Z. G. J. Lumin. 1975, 11, 1

(50) Novoderezhkin, V. I.; Salverda, J. M.; van Amerongen, H.; van Grondelle, R. J. Phys. Chem. B 2003, 107, 1893.

(51) Novoderezhkin, V. I.; Palacios, M. A.; van Amerongen, H.; van Grondelle, R. J. Phys. Chem. B 2004, 108, 10363.

(52) Macpherson, A. N.; Liddell, P. A.; Kuciauskas, D.; Tatman, D.; Gillbro, T.; Gust, D.; Moore, T. A.; Moore, A. L. J. Phys. Chem. B 2002, $106,9424$.

(53) Köhler, A.; Beljonne, D. Adv. Funct. Mater. 2004, 14, 11.

(54) Spano, F. Ann. Rev. Phys. Chem. 2006, 57, 217. 